Biologisches Institut der Universität Stuttgart

\title{
Die Fettsäurezusammensetzung der verseifbaren Lipide aus Rinden von Populus balsamifera im Jahresgang
}

U. Kull und K. Jeremias

Eingegangen am 21. Mirz 1972

\section{Summary}

The fatty acid composition of the saponifiable lipids from barks of Populus balsamifera during a year

1. The fatty acid patterns were investigated qualitatively and quantitatively by gas liquid chromatography in monthly intervals. The contents of fatty acids were evaluated as percentage of total fatty acids (shown in table 1) and as percentage of dry weight of barks (table 2).

2. Seventeen different fatty acids were found, twelve of them could be measured quantitatively during the whole year. The most important compounds are palmitic, oleic, linolic, and linolenic acid; myristic, myristoleic and behenic acid are found in considerable amounts, too. During spring another acid is predominating, which was tentatively ident1fied as margarinic acid.

3. During winter the contents of linolenic and especially of linolic acid are raised, the latter being the predominating acid during this season. The amounts of palmitic acid (in ${ }^{\circ} 0$ of dry weight) shows only slight variations in the course of the year. Expressed as " " of total fatty acids it has a minimum in winter.

4. The findings are discussed in relation to the influence of temperature on storage of lipids and on biosynthesis of fatty acids.

\section{Einleitung}

In höheren Pflanzen werden häufig außer Kohlenhydraten auch Lipide als Reservestoffe gespeichert. Außerdem sind Lipide wichtige Strukturbausteine in allen pflanzlichen Zellen. Ihr physiologisches Verhalten wurde mit modernen Methoden bisher vor allem an Früchten und Samen untersucht. Uber die Verhältnisse in vegetativen Pflanzenteilen gibt es eine größere Anzahl älterer Arbeiten (vgl. ZELLer, 1957). Jedoch liegen bisher kaum neuere, mit gaschromatographischen Methoden gewonnene Befunde über Veränderungen der Fettsäurezusammensetzung von Lipiden im Jahresgang vor. Bei Pappeln ist eine winterliche Anhäufung von "Fett» zuerst von IsHiBE (1935) nachgewiesen und dann von JEREMIAS (1968 b) genauer geprüft worden. In Fortsetzung dieser Untersuchungen über die Mengenveränderungen des Gesamtlipidgehaltes in den Rinden von Pappelsorten wird hier über die Fettsäurezusammensetzung des verseifbaren Anteils dieser Lipide und ihre qualitative und quantitative Veränderung im Verlaufe eines Jahres berichtet. 


\section{Material und Methoden}

\section{Material}

Untersucht wurden Rinden des Balsampappelbastards Populus balsamifera L. Sorte Rochester in monatlichen Abstanden. Die systematische Stellung der Hybride ist bei JEREvis $(1968$ a) geschildert. Uber die Jahresperiodik der Reservekohlenhydrate, des * Rohfertes" und der "gebundenen" Lipide in den Rinden berichtet Jerrmias (1968 a, b).

\section{Methoden}

a) Die Extraktion der Lipide erfolgte mit Chloroform-Methanol nach dem Verfahren von WINTFR (1963), das schon von BFRINGER (1966) auf pflanzliches Material angewendet worden war. Wic bereits fruhere Untersuchungen (KuLL, $1972 \mathrm{a}, \mathrm{b}$ ) gezcige hatten, liegen dic Rohfett-Gehalte bei dieser Methode stets höher als bei der von Jerfmias (1968 b) verwendeten Extraktion mit Petrolather nach ZIRM (ZIRM et al., 1956). Dagegen sind die Werte für die nach Kochen mit Alkohol zusatzlich extrahierbaren "gebundenen" Lipide erheblich geringer und liegen bes Populus balsamifera in der Regel unter $5^{\circ}$ " der Gesamtlipidmenge (KuLl., 1972 b). Sie sind daher in dieser Arbeit unberuicksichtigt geblicben.

Soll anschließend eine gaschromatographische Trennung von Fettsaureestern erfolgen, so ist die schonendere Methode nach WINTFR vorzuzichen, da sie weitgehend Gewahr bietet, daß3 auch bei wenig stabilen Fettsïuren keine Strukturverinderung erfolgt. Allerdings ist bei den Rinden der Balsampappel ein unerwunschter Nebeneffekt zu beobachten: die harzartigen Stoffe des .Balsams" werden - im Gegensatz zur Petrolathermethode - in großem Ausmaß mir extrahiert und führen zu sehr hohen Gehalten nichtverseifbarer Stoffe in der Gesamtlipidfraktion. Daher sind Rohfettgehalte, die nach diesem Verfahren ermittelt werden, ziemlich hoch und infolge eines Anteils fluchtiger Verbindungen im Balsam mit Unsicherheir behaftet. Auf die Untersuchung der Fettsaurezusammensetzung des verseifbaren Anteils durfte jedoch dieser Umstand nach unseren Erfahrungen keinen nennenswerten Einfluß haben.

b) Die Verseifung der Lipide und Herstellung der Methylester erfolgte in einem Arbeitsgang in Anlehnung an die Methode von STOFfFl et al. (1959; vgl. BAyER, 1962). Dabei wurde stets unter strömendem Stickstoff gearbeitet. Daten der Gaschromatographie: Gerät VarianAerograph 1840-4; Saule 20' 1/8" Stahl, $10^{\prime}{ }^{\prime}$. EGSS-X auf 60-80 Chrom WAW-DMCS; Saulentemperatur: 180 C; Detektor: FID, 260 C; Injektortemperatur: 210 C; Tragergas: Stickstoff, normalerweise $30(\mathrm{ml} / \mathrm{min})$.

c) Die Identifizterkng der einzelnen Peaks erfolgre durch Bestimmung der relativen Retentionsvolumina und Mitchromatographieren von Bezugssubstanzen unter Variation von Trägergasdruck und Säulentemperatur. Als Bezugssubstanzen standen zur Verfügung die Methylester von (in Klammern jeweils die in den Tabellen verwendete Abkürzung): Caprinsaure $(10: 0)$, Laurinsäure (12:0), Lauroleinsaure (12:1), Myristinsaure (14:0), Myristoleinsaure (14:1), Pentadecansaure $(15: 0)$, Palmitinsäure $(16: 0)$, Palmitoleinsäure (16:1), Margarinsảure (17:0), Stearinsäure (18:0), Olsaure (18:1), Linolsäure $(18: 2)$, Linolensaure $(18: 3)$, Arachinsiure $(20: 0)$, Behensäure $(22: 0)$. Herkunft: Fa. Merck, Darmstadt, sowie Testmischungen der Applied Science Lab. State Coll., Pennsylvania.

d) Die quantıtatıve Mengenbestımmung erfolgte durch Berechnung der Peakflächen mit dem Naherungsverfahren Peakhöhe $\times$ Halbwertsbreite (vgl. KaISER 1965). Als innerer Standard diente der Methylester der Pentadecansäure, da diese nur in geringen Spuren im Pflanzenmaterial aufzufinden war. Eine Korrektur erfolgte bei längerketrigen Fettsäuren (ab Olsaure) mit Hilfe von Eichwerten, die unter Anwendung quantitativer Testgemische (der Appl. Sci. Lab.) erhalten wurden.

Das Ausgangsmaterial wurde jeweils mindestens zweimal getrennt aufgearbeitet, jedes der dabei erhaltenen Methylestergemische 3-6 mal gaschromatographisch getrennt und die Flä- 
chen ermittelr. Die erhaltenen Mefiwerte wurden nach Bezug auf den inneren Standard gemittelt.

\section{Ergebnisse}

a) Qualitatice Zusarmmensetzung der Fettsüurefraktion

In den Rinden sind das ganze Jahr über alle geradzahligen gesattigten Fettsäuren von Caprinsäure bis Behensäure nachzuweisen. Zwei Peaks mit höheren Retentionswerten als Behensäureester konnten nicht identifiziert werden. Auch der Peah des Behensäureesters selbst enthält noch eine weitere Komponente, möglicherweise eine ungesättigte $\mathrm{C}_{20}$-Säure. Von den ungesättigten Fettsauren sind Myrristoleinsäure, Olsäure, Linolsäure und Linolensäure stets und zum Teil in großen Mengen nachzuweisen. Eine Hexadecensäure ist bei den für die quantitativen Untersuchungen verwendeten Empfindlichkeiten nur im Herbst meßbar, bei Erhöhung der Empfindlichkeit aber in sehr geringen Prozentsätzen (unter $0,05^{\circ}$ o der Gesamtfettsäuren) immer zu erkennen.

Ungeradzahlige Fettsäuren mit 13,15 und 19 C-Atomen konnten insbesondere in den Winter- und Frühjahrsproben in ähnlich geringen Anteilen nachgewiesen werden. Drei weitere nicht identifizierte Peaks mit kleineren Retentionsvolumina als Olsäure fallen mengenmäßig kaum ins Gewicht, sind aber vom Winter bis zum Spätfrühjahr stets vorhanden.

Der Methylester einer weiteren Hauptkomponente, welche während der Zeit von Februar bis Juni vorherschend wird, besitzt denselben Retentionswert wie Margarinsäureester. Durch Veränderung der gaschromatographischen Parameter konnte keine Abtrennung von authentischem Margarinsäuremethylester erreicht werden. Da es möglich erscheint, daß eine verzweigtkettige Fettsäure vorliegt, wurde ein Vergleich mit dem Fettsäuremuster von Abies-concolor-Nadeln durchgeführt. In Abies sind eine verzweigtkettige $C_{17}$-Fettsäure sowie mehrere ungesättigte $C_{16}$-Säuren nachgewiesen (Jamieson u. Reid, 1972). Durch Vergleich der relativen Retentionsvolumina und Kochromatographie erwies sich die fragliche Verbindung aus der Pappelrinde mit keiner der Fettsäuren aus Abies identisch. Die Fettsäure aus Populus wird daher als Margarinsäure (?) bezeichnet.

\section{b) Jahresperiodik der Fettsäuren}

Der Gesamtgehalt an verseifbaren Lipiden zeigt ein ähnliches Verhalten, wie es von Jeremias (1968 b) für das Gesamtfett und für das Rohfett gefunden worden war. Die langsame Mengenabnahme im Frühjahr entspricht dabei mehr dem damals bei der Sorte "Androscoggin" als dem bei "Rochester" gefundenen Verlauf.

Die Mengen der einzelnen Fettsäuren sind im Prozent der Gesamtfettsäuren des Verseifbaren in der Tabelle 1 und in Prozent des Trockengewichts in der Tabelle 2 angegeben.

Von den mengenmäßig wichtigeren Fettsäuren zeigt Palmitinsäure im Jahresgang nur geringfügige Schwankungen des Absolutgehaltes um den Mittelwert bei etwa $0,19 \%$ des Trockengewichtes. Deutlich höhere Werte treten nur in den Herbstmona- 


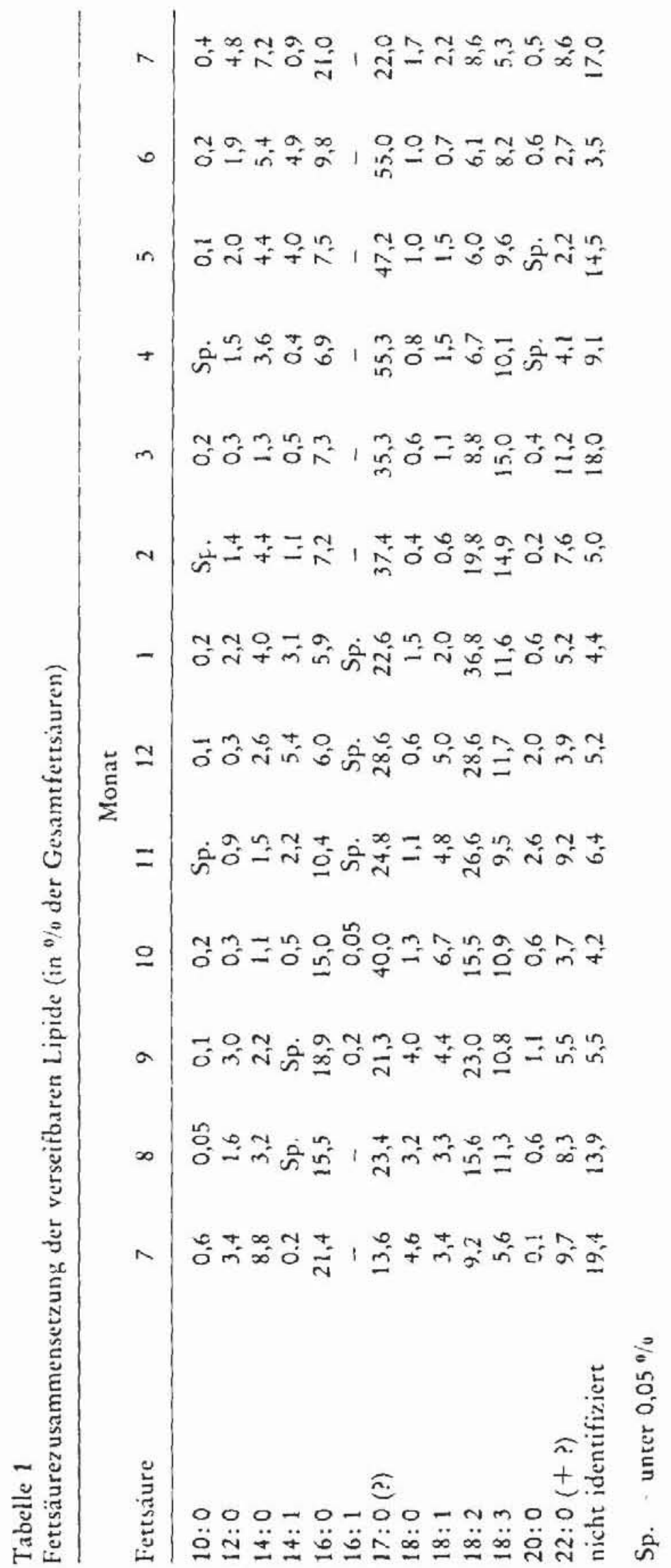

Z. Pflanzenphystol. Bd. 68. S. 55-62. 1972. 


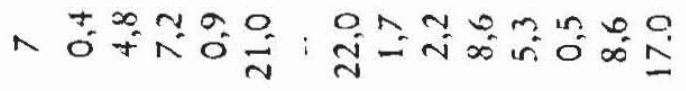

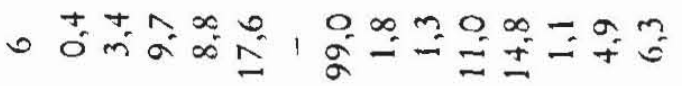

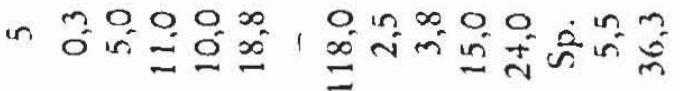

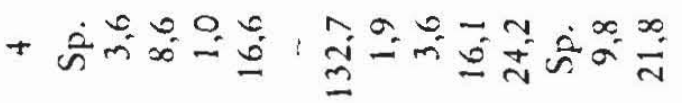

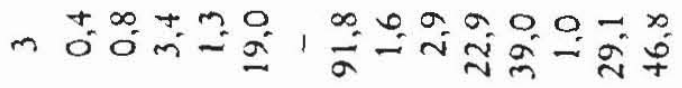

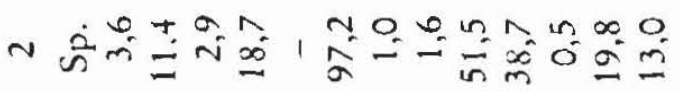

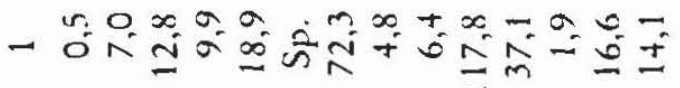

Е

$\stackrel{5}{\Sigma}$

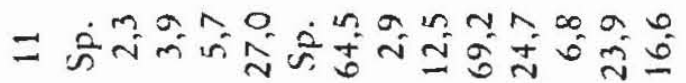

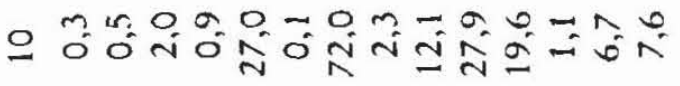

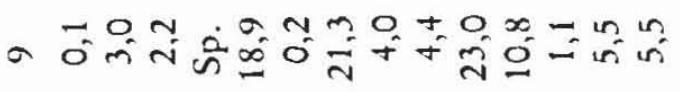

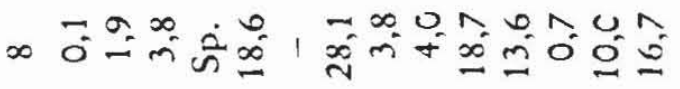

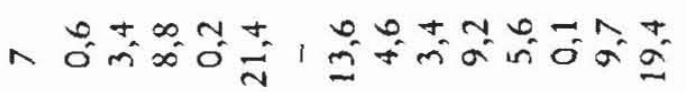


ten auf. Ihr prozentualer Anteil an den Fettsäuren ist somit in den Sommermonaten am höchsten, nimmt zum Winter hin ab und steigt erst im Juni/Juli wieder an. Bei der Margarinsäure (?) ist die Absolutmenge im Sommer am gerinsten. Ein unregelmäßiger Anstieg im Herbst und Winter führt zum Gehaltsmavimum im April. Dann erfolgt bis Juli eine rasche Mengenabnahme. Beim prozentualen Anteil ergibt sich ein kleineres Maximum im Oktober und ein ausgeprägtes Frühjahrsmaximum. In der Zeit von Februar bis Juni ist diese Substanz die vorherrschende Fettsäure und erreicht Anteile bis zu über 50" o der Fettsäuren.

Große Bedeutung haben ferner Linol- und Linolensäure. Während der Wintermonate ist erstere mengenmäßig und im prozentualen Anteil die wichtigste Fettsäure überhaupt. Linolensäure zeigt ab Herbst einen langsamen Mengenanstieg bis zu einem Maximalwert im März mit 0,39 'o des Trockengewichts. Zum Sommer hin findet wieder eine Abnahme statt. Der prozentuale Linolensäuregehalt ist während des ganzen Jahres ziemlich konstant; nur im Spätwinter ist eine schwache Zunahme zu erkennen.

Die Menge an Myristoleinsäure steigt bis auf über 5 "0 der Gesamtfettsäuren an. Absolute und prozentuale Maximalwerte werden im Dezember und im Frühjahr erreicht. Ober $5 \%$ des Fettsäureanteils besitzt während der Herbstmonate ferner die Olsäure.

Auffällig ist die zeitliche Abfolge der absoluten Maximalgehalte der wichtigsten geradzahligen Fettsäuren: sie liegen bei Palmitinsäure im Oktober/November, bei Olsäure im Dezember, bei Linolsäure im Februar und bei Linolensäure im März.

Die nicht identifizierten Verbindungen machen während des Winters prozentual um $5^{\circ} \%$ aus, im Sommer dagegen bis zu $20^{\prime \prime}$. . Die daraus errechneten Absolutmengen liegen in den Wintermonaten bei $0,15 \%$ des Trockengewichts und steigen im Frühjahr stark an. Vermutlich steht dies mit der Balsam-Bildung im Bereich der Knospen im Zusammenhang.

\section{Diskussion}

Auffällig ist die relativ großse Zahl verschiedener Fettsäuren, die in nennenswerten Mengen in den Lipiden gefunden wurden. Acht Verbindungen sind zumindest in einem Monat mit über $5 \%$ Anteil vertreten, 6 weitere mit über $0,05 \%$. Drei Fettsäuren mit einem Anteil unter $0,05 \%$ konnten identifiziert werden. Außerdem besitzen 5 unbekannte Verbindungen Gehalte von zusammen bis nahe an 20\%. Im Verlauf unserer Untersuchungen zeigte sich allerdings, daß die Rinde von Populus balsamifera infolge ihres hohen Gehaltes an Unverseifbarem für die Bearbeitung des Lipidhaushaltes kein optimales Versuchsobjekt ist.

Die Zunahme des Lipidgehaltes und somit auch der daran beteiligten Fettsäuren in der kalten Jahreszeit ist schon von Jeremsas (1968 b) als Temperatureffekt gedeutet und diese Erklärung durch zusätzliche Versuche erhärtet worden. Dies steht mit früheren Befunden (vgl. Zeller, 1957) im Einklang. Die Angabe von Meara (1957), 
wonach in Rinden vorwiegend Olsäure gespeichert werde, trifft für die Pappel sicher nicht zu.

Aus vielen Untersuchungen an Früchten und insbesondere an Samen ist bekannt, daß Verringerung der Wachstumstemperatur zu einer Zunahme der Jodzahl der Lipide führt (z. B. Dybing; u. Zimmerman, 1966; vgl. Zelleer, 1957; Mizlis 1968). Neuere Arbeiten unter Anwendung der Gaschromatographie haben gezeigt, daß hierbei in der Regel eine Mengenzunahme mehrfach ungesattigter Fettsäuren stattfindet (Canin, 1965; Rinive, 1969; Beringer, 1971 a; Schuster, 1971). Für Lipide aus Algen ist ein entsprechender Temperatureinfluk nachgewiesen worden (Holtox et al., 1964; P.tterson, 1970). Bei Angiospermen liegen Untersuchungen über die Veränderung der Fettsäurezusammensetzung von Lipiden aus Blättern und Wurzeln bei der Kältehärtung der Luzerne vor (Gerloff et al., 1966; Kilipf.r, 1970). Auch hier ist eine gleichartige Wirkung der Temperatursenkung zu beobachten.

Unsere Befunde über die Gehaltsveränderungen ungesättigter Fettsäuren, insbesondere der mengenmäßig bedeutsamen Linol- und Linolensäure, stehen damit in Einklang. Somit ist auch für Rindengewebe einer Holzpflanze eine temperaturbedingte winterliche Vermehrung mehrfach ungesättigter Fettsäuren nachgewiesen. Im Gegensatz zu den bisher besprochenen Arbeiten fanden ZIRM et al. (1956) in den immergrünen Blättern von Efeu und Rhododendron bei ansteigendem Lipidgehalt im Winter eine Abnahme der Jodzahl und der Zahl konjugierter Doppelbindungen in der Lipidfraktion. Eine Uberprüfung dieser Ergebnisse mit gaschromatographischen Verfahren wäre wünschenswert.

Bei Untersuchungen über die Temperaturabhängigkeit der Biosynthese von Fettsäuren konnten HARRIS und JAMEs (1969) an Samen mehrerer Arten sowie RINNE (1969) an keimenden Sojabohnen eine verstärkte Bildung ungesättigter Fettsäuren zuungunsten gesättigter bei tiefer Temperatur nachweisen. Zwar lassen sich aufgrund unserer Daten keine unmittelbaren Aussagen über den Umsatz der Fettsäuren machen, aber die Anhäufung der ungesättigten Säuren während der kalten Jahreszeit läßt entsprechende Vorgänge wahrscheinlich erscheinen. Demgegenüber bleibt bei der Pappel der Gehalt an Palmitinsäure weitgehend konstant. Dies kann mit dem Befund von RINNE (1969), wonach die Biosynthese dieser Verbindung (bei der Sojabohne) durch die Temperatur nicht beeinflußt wird, verglichen werden.

Interessant erscheint ferner die zeitliche Abfolge der Gehaltsmaxima der mengenmäßig wichtigeren geradzahligen Fettsäuren in den Wintermonaten. Sie entspricht den bekannten Wegen der Biosynthese der Fettsäuren in höheren Pflanzen (vgl. z. B. NAGAI u. BLOCH, 1966; STLMPF, 1970). Da wir über den Umsatz nichts aussagen können, muß offen bleiben, ob hierbei gespeicherte Palmitin- bzw. Olsäure als Vorstufen der mehrfach ungesättigten Fettsäuren fungieren (vgl. Beringer, 1971 b).

Uber Beziehungen des Lipidhaushaltes, und insbesondere der Vermehrung mehrfach ungesättigter Fettsäuren, zu Kältehärtung und Kälteresistenz der Pflanzen ist seit dem vorigen Jahrhundert immer wieder berichtet worden (LEvirt, 1956; vgl. KUIPER, 1970). Neue Untersuchungen über Phasenumwandlungen in Lipiden (Trä̈U- 
BLE, 1971) machen es wahrscheinlich, daß die Fettsäurezusammensetzung der Lipide zellulärer Membranen auf dic Kälteresistenz von erheblichem Einfluß sein kann. Verseifbare Lipide treten in der pflanzlichen Zelle aber nicht nur als Membranbausteine, sondern auch als Reservestoffe auf. Unsere Untersuchungen lassen nicht erkennen, welchen Anteil diese verschiedenen Komponenten haben. Daher erscheint eine genauere Diskussion dieses Problemk reises nicht sinnvoll.

Für die Oberlassung des Versuchsmaterials danken wir Herrn Reg.-Dir. Dr. G. Sc.HLfNkfR von der Baden-Wurttembergischen Forstlichen Versuchs- und Forschungsanstalt. Fr.iulein SiEc;LINDE VON KIR gilt unser Dank für dic sorgfaltige Aufarbeitung und Vorbereitung der Proben für die Gaschromatographie. Einer der Autoren (U. K.) dankt der Deutschen Forschungsgemeınschaft für eine Sachbeihilfe.

\section{Literatur}

Bayfr, E.: Gas-Chromatographie, 2. Aufl., Springer-Verlag 1962.

Brringer, H.: Z. Pflanzenernihrg., Düng., Bodenkde. 114, 117 (1966).

- Z. Pflanzenernahrg., Düng., Bodenkde. 128, 115 (1971 a).

- Plant Physiol. $48,+33$ (1971 b).

Canvin, D. T.: Canad. J. Bot. 43, 63, (1965).

Dybing, C. D., and D. C. Zimmerman: Plant Physiol. 4l, 1465 (1966).

Gerloff, E. D., T. Richardson, and M. A. Stahmann: Plant Physiol. 41, 1280 (1966).

Harris, P., and A. T. James: Biochim, biophys. Acta 187, 13 (1966).

Holton, R. W., H. H. Blecker, and M. Onore: Phytochemistry 3, 595 (1964).

Ishibe, O.: Mem. Coll. Sci. Kyoto Imp. Univ., Ser. B, 11, 1 (1935).

Jamieson, G. R., and E. H. Reid: Phytochemistry /l, 269 (1972).

JrR rmias, K.: Mitt. Ver. forstl. Standortskde. u. Forstpfl.-züchtung 18, 89 (1968 a).

- Mitt. Ver. forstl. Standortskde. u. Forstpfl.-zuchtung 18, 95 (1968 b).

KaISER, R.: Chromatographie in der Gasphase, Teil IV, Bibl. Inst. Mannheim 1965.

Kuiper, P. J. C.: Plant Physiol. 45, 684 (1970).

KuLl, U.: Ber. dtsch. bot. Ges. 84, 299 (1972 a).

- Bot. Studien (Jena) 19 (1972 b).

Levitt, J.: The hardiness of plants (Agronomy, Vol. VI) Acad. Press. 1956.

Mazliak, P.: Le métabolisme des lipides dans les plantes superieures. Masson, Paris 1968.

Mrara, M. L.: Handbuch der Pflanzenphysiologie, ed. W. Ruhland et al., 7, 10 (1957).

Nagal, J., and K. Bloch: J. biol. Chem. 241, 1925 (1966).

Patterson, G. W'.: Lipids S, 597 (1970).

Rinne, R. W.: Plant Physiol. 44, 89 (1969).

Schister, W.: Fette, Seifen, Anstrichmittel 73, 305 (1971).

Stoffel, W. F., and E. H. Ahreis, jr.: Analyt. Chem. 31, 307 (1959).

Stumpf, P. K.: Comprehensive Biochemistry, ed. M. Florkin and E. H. Stotz, 18, 265 (1970)

Trauble, H.: Naturwissensch. 58, 277 (1971).

WINTER, E.: Z. Lebensmittel-Unters. u. Forsch. 123, 205 (1963).

Zelifr, A.: Handbuch der Pflanzenphysiologie, ed. W. Rlihland et al., 7, 280, (1957).

Zirm, K. L., A. Pongratz and W. Polesofsky: Z. Naturforschg. 11 B, 110 (1956).

Doz. Dr. U. Kutl, Prof. Dr. K. Jeremias, Biologisches Institut der Universität, D-7000 Stuttgart 60, Ulmer Straße 227.

Z. Pflanzenphystol. Bd. 68. S. 55-62. 1972. 